\title{
IV: TEXTGRUNDLAGE UND ÜBERSETZUNG
}

Die Textgrundlage des Bremer Evangelistars ist wie im Mittelalter üblich die Vulgata ${ }^{1}$. Nur hier und da sind möglicherweise Lesarten der Vetus Latina ${ }^{2}$ als Vorlage der Übersetzung anzunehmen:

Lk 2,20 - Vulgata: sicut dictum est ad illos

Vetus Latina: sicut Dominus ostendit illis $\beta$

12,10: alse in gezouget waz $\mathrm{Fr}$

alse in geseit waz $\mathrm{Br}$ als in gesaget was $\mathrm{Nl}$ als in gesait waz $\mathrm{N2}$

Lk 5,25 - Vulgata: confestim surgens coram illis tulit in quo iacebat

Vetus Latina: confestim surgens coram illis (sus)tulit lectum $b \boldsymbol{r}^{l}$ [grabattum (suum $a) a d$ ]

continuo surrexit in conspectu eorum et tulit grabattum suum $A$ fra

124,18: der betteresige hub uf sin bette Da er anelag. Fr er hup of daz bette da er offe lag $\mathrm{Br}-\mathrm{N1}$ der pettrise hub auf daz pett da er an lag N2

Mt 21,23 - Vulgata: ... accesserunt ad eum docentem principes sacerdotum ... Vetus Latina: ... accesserunt ad eum principes sacerdotum ...

136,2: Da gingen zu yme die fursten der pristere. Fr do gingen zù ime die fursten. $\mathrm{Br}$ do gingen $\mathrm{zu}$ im die fursten der prister $\mathrm{N2}$

1 Zitiert nach: Biblia Sacra iuxta vulgatam versionem adiuvantibus BONIFATIO FISCHER e. a. recensuit et brevi apparatu instruxit ROBERTUS WEBER editio altera emendata, tomus II, Stuttgart 1975.

2 Zitiert nach: Itala. Das Neue Testament in altlateinischer Überlieferung, hrsg. von ADOLF JǓLuCHER, Bd. I: Matthäus-Evangelium, 2. Aufl. Berlin 1972; II: Marcus-Evangelium, 2. Aufl. Berlin 1970; III: LucasEvangelium, 2. Aufl. Berlin 1976; IV: Johannes-Evangelium, Berlin 1963. 
Allerdings ist der hier angenommene Einfluß der Vetus Latina nicht zwingend. Im ersten Fall könnte eine Assoziation der Stelle kurz zuvor et ostendit nobis $(\mathrm{Lk} 2,15)$ 'vnde vns irzouget ist' $(12,4) \mathrm{zu}$ der abweichenden Übersetzung geführt haben. In den beiden anderen Fällen handelt es sich um einen Zusatz bzw. eine Auslassung, die nicht notwendigerweise eine andere Textgrundlage erfordern. Ob die drei oben erwähnten $\mathrm{Fälle}^{3}$, in denen die Vetus Latina usque in finem - die Afrikanische Version usque ad finem - gegenüber in finem der Vulgata überliefert, hier anzureihen ist, bleibt ebenso fraglich. Da das Bremer Evangelistar nicht im Stil einer Interlinearversion vorgeht, genügt nämlich der Wortlaut der Vulgata, um die hier vorliegende Wiedergabe mit 'bit' zu erklären.

Hinsichtlich der Textgrundlage sind jedoch die nichtbiblischen Zusătze von größerem Gewicht, da sie gewisse Rückschlüsse auf das geistige Umfeld dieses Perikopenbuches ermöglichen. Sie finden sich in den Perikopen 1 (Fr Br N1) und 35 (Br; - Fr N1 N2) sowie gehäuft in der Passionsharmonie (89: Fr), die aus dem Übersetzungszweig des 'Leben Jhesu' stammt. In der folgenden Zusammenstellung werden jeweils nach diesen wie in der Edition durch ausgeblendete Schrift hervorgehobenen Passagen entsprechende Parallelstellen aufgeführt, soweit sich solche ermitteln ließen. Inwieweit diese von nahezu wörtlicher Übereinstimmung bis zu entfernteren Anklängen reichenden Entsprechungen bzw. die Vorlagen oder Quellen dieser Entsprechungen unmittelbare Vorlagen für das Bremer Evangelistar gewesen sind, dürfte sich kaum entscheiden lassen.

1. Perikope 2, Z. 3-5 (eingeschoben in Mt 11,2):

Da santus iohannes predigete $\mathrm{Da}$ in wolde got nicht predigen. also schire alz Johannes wart gefangen Da begunde der heillant zu predigen.

2. Perikope 35, Apparat zu Z. 8 (zu Mt 11,28):

komet zu mir alle die da arbeyden vnde gesunt sint. Das ist also vil gesprochen. Die da arbeydent in deme dinste gotes die gesuntheit betudet Daz ein ygelich mensche sal huden sich vor allir sunde. Daz iz ane sunde sy. So iz der dot begriffet dotlich odir degelich. So gefellet iz gote wole.

3. Perikope 89 , Z. 238 (zu Jo 18,40 ):

vf die zijt waz eyner gefangen der hiez barrabam vnde waz eyn ubiltediger mensche gewest vnde ein morder vnde hatte den dot. virschuldiget.

3 Vgl. oben S. LXII (Jo 13,1 - 88,5) und S. LXIII (Mt 10,22 - 202,11; MK 13,13 - 212,22). 
... / ainen der ain menschen tódt hatt / mit namen Barrabam / wôlt jr das ich euch ledig laß disen manschlechtigen / wirdig des tods / ...4

4. 89 , Z. 249f. (zu Mt 27,21):

Da rieffen sie myt eyme gemeynen munde. barrabam salt du lazsen gan. vnde ihesum an eyn cruce han.

Aminadab: Barrabam sollt du lassen gan, Jesum sollt du Crützgen lan.'

Sinagogus dicit: Barrabam, den laß uns gan, Jhesum saltu uns crutzen lan! ${ }^{6}$

Sinagoga dicit: Herre, du salt mit uns gan und Jhesum an eyn crucz han! ${ }^{7}$

5. 89, Z. 260f. (zu Mt 27,26$)$ :

Da liez pylatus barrabam vnde ihesum det her geyseln vnde an eyne sul byndden. vnde wart geslagen also sere Daz yme nye nicht gantzes an syme gotlichin lybe bleyb

Do ward du gegeyselt das nichs gancz belayb an allem deinem heiligen leichnam ... vnd punten dich vmb ein grosse sewl ...8

6. 89 , Z. 267 (zu Mt 27,29 ):

... vnde flachten yme eynen cronen von dornen vnde sasten sie yme. vf syn hobet. vnde sasten in uf eynen stul. vnde gaben ym ein ror in syne hant.

... vnd seczten dich auf ein stul ...9

4 Das Evangelium Nicodemi in spãtmittelalterlicher deutscher Prosa. Texte, hrsg. von ACHMM MAssER / MAX SILLER, Heidelberg 1987, S. 474 (Z. 270-273) \{Deutscher Druck. Dazu vgl. ebenda, S. 107ff.\}.

5 Das Luzerner Osterspiel, hrsg. v. HeInz WYss, Bd. II: Text des zweiten Tages, Bern 1967, S. 157.

6 Das Frankfurter Passionsspiel von 1493. In: Das Drama des Mittelalters, hrsg. von Richard FronING, Stuttgart 1891/92, Nachdruck Darmstadt 1964, S. 496.

7 Das Alsfelder Passionsspiel, in: Das Drama des Mittelalters (wie Anm. 6), S. 721. Hier nur wegen des zweiten Verses angeführt.

8 Hardo HIrG, Das Marienleben` des Heinrich von St.Gallen. Text und Untersuchung, München 1981, S. 259 (Kap. XV, 438, Lesart $X_{2}$ und 441).

9 HARDo HIIG (wie Anm. 8), S. 260 (Kap. XV, 448f.). 


\section{LXVIII}

7. 89 , Z. 312 (zu Jo 19,24):

sye hant geteylet min gewant. vnde vm myn gewant hant sie daz loz gewurffen. Dit taden die rittir die ihesus huten in dem grabe. ${ }^{11}$

\section{89, Z. 345 (zu Mt 27,34):}

eyner ... nam eynen swam ... vnde fulte yn mit ezsige vnde myt gallen vnde gap yme zu drincken vnde er der gallen gewar wart $\mathrm{Da}$ wolde er nicht drincken. want in dorste nach dem heyle dez menschin

alß dich gedurst hat $\left[X_{2} \sim\right.$ hat gedürst $]$ noch meinem hail vnd aller menschen $\left[X_{2}:+ \text { hayl }\right]^{12}$

9. 89 , Z. 352 (zu Mt 27,53):

... vnde vil der toden gingen $\mathbf{v z}$ den grebern. vnde bewisten sich den luden nach syner vffirstende in der heilgen stat zu gezugnuzze syner gotlichin warheit.

10. 89, Z. 354 (zu Mt 27,54):

Da stunt da neben deme cruce eyn man der waz edil vnde hiez zenturius. Der hatte hundirt ritter vndir yme.

Centurio + qui centum milites sub se habet...$^{13}$

Centurio dicitur ille, qui habet centum milites sub $\mathrm{se}^{14}$

der was geheissen Do Centurio, wan er hatte vnder im hundert ritter ... ${ }^{15}$

11. 89 , Z. 373 (zu Jo 19,34):

Sủndir der rittir eyner vffinte yme syne siten myt eyme sper. zuhant floz daruz wassir vnde blut vnde der rittir hiez longinus vnde waz blynt vnde wart gesehin.

vnd der Ritter Longinus stach im ein sper in sein seytten, doraus ran wasser vnd plut. der was plint, der straich das an seine augen vnd ward dauon gesehent. ${ }^{16}$

11 Das Leben Jhesu, hrsg. von Christoph GerhardT, Leiden 1970, S.161, Z. 9f., hat nur die auf Mt 27,36 et sedentes servabant eum gestützte Passage: dise waren bch die sin hôten do er an dem krüce hieng.

12 HARDO HIIG (wie in Anm. 8), S. 271 (Kap. XV, 668f.).

13 Summarium Heinrici, hrsg. von ReINER HIIDERRANDT, Bd. II, Berlin 1982, S. 218.

14 Vocabularius Ex quor. Überlieferungsgeschichtliche Ausgabe. Gemeinsam mit KLAUS GrUBMÖLER hrsg. von BERNHARD SCHNELL u. a., Bd. II, Tübingen 1988, S. 482.

15 Das Evangelium Nicodemi (wie Anm. 4), S. 267 (Z. 490) \{Fassung E\}.

16 Das Evangelium Nicodemi (wie Anm. 4), S. 234 (Z. 239f.) \{Fassung D\}. 
12. 89 , Z. 379 (zu Mt 27,57):

Du quam eyn richir man vnde edel. Der zehin rittir hatte vndir yme Der waz von arromatia der stat in iuda ein Jůde. ${ }^{17}$

Neben Erläuterungen in Form von Glossierungen wie Nr. 10 und Nr. 12 handelt es sich um nähere Angaben zum Passionsgeschehen, die im biblischen Bericht nicht enthalten sind: Die Todeswürdigkeit von Barabbas (Nr. 3), die Geißelsäule (Nr. 5), der Thron im Zusammenhang der Verspottung Jesu (Nr. 6), die Identität der Soldaten, die um das Gewand des Gekreuzigten würfeln, mit den Grabeswächtern (Nr. 7) und Longinus ${ }^{18}$ als Name des Soldaten mit der Lanze, seine Blindheit und wundersame Heilung (Nr. 11). Vor allem durch die Passionsliteratur, die Predigt, das geistliche Spiel und bildliche Darstellungen waren diese Dinge - mit Ausnahme von der hier einzureihenden Nachricht über den verzögerten Beginn der Predigt Jesu (Nr. 1) - den spätmittelalterlichen Menschen geläufig. In dem reimenden Einschub (Nr. 4) ist der nachhaltige Einflu $B^{19}$ des mittelalterlichen Spiels unmittelbar zu greifen. Die Zusätze Nr. 5 (zweiter Teil), Nr. 8 und Nr. 9 sind von anderer Art. Sie deuten das Heilsgeschehen zwar nicht in gelehrt-theologischer Weise in der Tradition der Bibelkommentare ${ }^{20}$, sondern im Rahmen einer 'Theologia practica'21, wie dies für die Erbauungsliteratur des Spātmittelalters charakteristisch ist. In der Bremer Handschrift ist dieses Moment durch die zahlreichen eingeschobenen Kurztexte ${ }^{n}$ wesentlich verstärkt. Ebenfalls in diesen Zusammenhang gehört der nur dort überlieferte Zusatz (Nr. 2), der wohl durch ein Verlesen bzw. eine Verderbnis der Vorlage - 'gesunt' statt 'beswert' - ausgelöst worden ist. ${ }^{23}$ Den beiden Nürnberger Handschriften fehlt diese Einbettung; sie halten sich - mit Ausnahme von Nr. 1 in N1 - allein an den Bibeltext. Das besagt aber nicht, daß sich die Ge-

17 Der Zusatz steht so schon im Leben Jhesu (wie Anm. 11), S. 164, Z. 7. Vgl. auch unten S. 179, Z. 281.

18 Vgl. Hemron Alzhemmer, 'Longinus'. In: Lexikon des Mittelalters, Bd. 5, München / Zürich 1991, Sp. 2107.

19 Zur Wirkungsgeschichte vgl. HeInZ KINDERMANN, Das Theaterpublikum des Mittelalters, Salzburg 1980, besonders Kap. 3: Das Publikum der deutschsprachigen Passions- und Legendenspiele, S. 26ff.

20 So z.B. in den 'Melker Evangelien'. Vgl. JochEN SPLETT, Die Zuordnung zu Übersetzungszweigen. In: Vestigia Bibliae $9 / 10$ (1991), S. $42 \mathrm{f}$.

21 Vgl. Olaf SCHWENCKE, Strukturen des Spätmittelalters und deutsche Literatur. Eine Studie. In: CollGerm 1970, S. 174ff. Zum Thema allgemein sei verwiesen auf die einzelnen Beiträge im Sammelband 'Laienfrōmmigkeit im spāten Mittelalter', den KLAUS SCHREINER 1992 herausgegeben hat.

22 Soweit möglich mit Nachweis der Quellen- bzw. Paralleltexte hier im Nachtrag, S. 365-369, ediert.

23 Vgl. dazi auch WrLYY LODTKE, Evangelientexte, besonders aus Harmonien, Nürnberg 1965, S. 25, der allerdings von einem Übersetzungsfehler ausgeht, da ihm der Zusammenhang mit den übrigen Textzeugen nicht bekannt war. 
brauchssituation, über die diese Textzeugen keine direkten Aussagen machen, grundlegend verändert hätte.

Die Übersetzung ist schlicht und schlägt einen Mittelweg ein zwischen einer strikten Ausrichtung am Wortlaut des Ausgangstextes und einer sich davon gänzlich lösenden freien Wiedergabe. Sie ist nicht fehlerfrei, wobei allerdings zu berücksichtigen ist, daß es sich bei allen Handschriften um Abschriften handelt. Wieviel also den Abschreibern anzulasten ist und wieviel dem bzw. den Übersetzern, ist nicht immer eindeutig zu entscheiden. So entspricht etwa omnis vallis implebitur et omnis mons et collis humiliabitur (Lk 3,5) in den Handschriften (Perikope 9, 9-12):

Eyn ygelich dayt wirt irfullit vnde ein igelich werg irhohet. Odir ein bohil eyme igelichme. vnde wirt irfullet ein ygelich werg vnde ein ygelich bohel eyme iglichen wirt irfullet ein iglich behelder irfullet

wirt genyddert $\mathbf{F r}$ wirt genyddert $\mathrm{Br}$

genidert vnd ein iglich dal wirt irhöhet $N 1$

dayt 'Tat, das Tun' in Fr dürfte auf ein verlesenes dayl der Vorlage zurückgehen. Von da aus ist wohl berg zu werg 'Werk, Tat'24 umgedeutet worden; denn eine bloße graphische Umsetzung scheidet aus, da $w$ für anlautendes $b$ in dieser Handschrift nicht bezeugt und auch für die anzunehmende Vorlage nicht plausibel ist. ${ }^{25}$ So ergibt sich zwar kein sinnvoller Textzusammenhang, aber isoliert gesehen doch ein verständlicher Satz. In $\mathrm{Br}$ sind die beiden ersten Wörter zum unmittelbar vorangehenden vide machet syne fuzpede gerecht gezogen, dayt und irhohet weggelassen und auf diese Weise der Stelle ein wiederum anderer Sinn gegeben worden. In $\mathrm{N} 1$ ist zwar mit dem nachgestellten ein iglich dal wirt irhohet der lateinische Halbvers zutreffend wiedergegeben, aber in der Passage davor ist das syntaktische Gefüge gestört und durch den Ersatz von werg durch behelder 'Behältnis'26 die vorliegende Verderbnis nicht behoben.

24 Durchgehend in dieser Form in Fr als Übersetzung von opus bzw. opera $(2,2 ; 37,6 ; 52,15 ; 52,17 ; 57,7$; 67,$44 ; 71,12 ; 72,7 ; 72,8 ; 78,4 ; 78,8 ; 79,7 ; 79,14 ; 79,16 ; 82,7 ; 111,17 ; 111,19 ; 121,11 ; 121,12 ; 121,13$; 200,12) belegt.

25 Vgl. ROBert PEter Ebert u. a., Frühneuhochdeutsche Grammatik, Tübingen 1993, \& L 44,2, S. 85.

26 Die alte, im Frühneuhochdeutschen vorherrschende Bedeutung 'Erlöser, Erretter' liegt hier sicher nicht vor, zumal in N1 das in den anderen Handschriften bezeugte behaldir 'Erlöser, Erretter' $(67,57 ; 69,23)$ stets durch heilant bzw. heylant ersetzt wird. GrRMM, Deutsches Wörterbuch I, Sp. 1324, bringt als Erstbeleg 
Derartige Störungen der Überlieferung, bei denen ohne Rūcksicht auf den Bibeltext nur eine partielle, auf den einzelnen Satz beschränkte Stimmigkeit vorliegt, kommen vielfach auch in nur einer Handschrift vor wie beispielsweise in Perikope $146 \mathrm{zu} \mathrm{Lk} \mathrm{8,27} \mathrm{et} \mathrm{vesti-}$ mento non induebatur : Der nye nicht gewan Fr. Die anderen Textzeugen überliefern eine zwar nicht wörtliche, aber zutreffende Übersetzung: vnd in liez nicht gewandis an yme $\mathbf{B r}$ der liez nicht gewantz an im N2

Die unzutreffende Wiedergabe von vos autem dicitis quicumque dixerit patri vel matri munus quodcumque est ex me tibi proderit (Mt 15,5) ist dagegen nicht der Überlieferung anzulasten, sondern dem Übersetzer, wobei die mangelnde Vertrautheit mit den hier angesprochenen jüdischen Gebräuchen mit im Spiele war. Die Handschriften (Perikope 65, 7-9) überliefern (einschließlich der Wiedergabe des folgenden Verses):

Ir sprechent abir wer uatir odir mútir icht spreche Daz er etzliche gabe von in jch spreche aber das uwer fader vnd müder it spreche das ir Eicliche gabe von in jr sprechet aber wer vater oder múter icht sprichet daz er eicleiche gab von irn intphahe. Also habet ir eweclich gemacht daz gebot godes durch inpahent vnd ir hat alles vnrechte gedencke vnd vnrecht gemacht das gebode godes durch enphieng vnd also habt ir fppig gemachet daz gepót gocz durch

ewer gesecze $\mathrm{N2}$

uwir gesetze. Fr

uwer gesecze $\mathrm{Nl}$

In $\mathrm{Br}$ ist diese Perikope noch einmal mit nur minimalen Abweichungen als Nachtrag überliefert - beide Male von der 5. Hand geschrieben. Hier ist offensichtlich die unverständliche Wiedergabe bemerkt und durch eine erläuternde Passage ersetzt worden. Entnommen ist sie dem Übersetzungszweig des 'Leben Jhesu'27:

vnd ir twinget die kinder dar zu mit uwirn geboten daz sie moszen sagen irn fetern vnd irn motern myn gabe die ich an dich legen músz vmme dir zu helffen die sult ich geoppert haben vnd in diesem wise $\left({ }^{\circ} \mathrm{Br}^{\circ}\right)$ daz ir sie (+ da) twinget so dut ir sie brechen daz gebot gotes vmme uwir gesetze wan sie nit eren vater vnd moter sundir sie vneren sie mit vnvirwiszelichen worten vnd ... Br Br

für behalter im Sinne von 'Behältnis' eine Stelle aus der um 1460 entstandenen, ersten Decameron-Übersetzung, und zwar aus einem Straßburger Druck von 1519.

27 Vgl. oben S. LIVf. 
Viel hăufiger sind Auslassungen, die mehrere, einzelne oder Teile von Bibelversen umfassen. In den beiden ersten Fällen ist dies den Stellenangaben unter der Perikopennummer und der Liste der Bibelstellen ${ }^{28}$ zu entnehmen; im letzten Fall stehen entsprechende Angaben in den Anmerkungen. Zusätze bzw. Textänderungen aufgrund von Parallelstellen und Wiederholungen in derselben Perikope sind bei dieser Textsorte nichts Ungewöhnliches und finden sich auch hier, wie die folgenden Beispiele zeigen:

Die andern brachin este von den boumen. vnde strauweten sie an den weg $\mathrm{Fr} \mathrm{N2} 2^{29} \mathrm{zu}$ Mt 21,8 als Einschub in die Perikope 83 vom Einzug Jesu in Jerusalem nach Lk 19, 29-40.

Die da duben kouften $\mathrm{Fr} N 2^{30} \mathrm{zu}$ Jo 2,16 anstelle von nicht übersetztem vendentes in illo et ementes (Lk 19,45) in der Perikope 151 von der Weissagung über Jerusalem und Jesus im Tempel nach Lk 19, 41-47.

Gent von mir ir virfluchten. Jn daz ewege fur. $\mathrm{Fr} \mathrm{Br} \mathrm{N1} \mathrm{N2^{31 }} \mathrm{zu}$ Mt 25,41 anstelle des nicht übersetzten discedite a me omnes operarii iniquitatis ( $\mathrm{Lk} 13,27)$ in der Perikope 157 vom Gleichnis von der verschlossenen Tür nach Lk 13, 22-29.

odir wen suchistu $\mathrm{Fr} B r \mathrm{N1} N 2(96,9)$ zu Jo 20,15 nochmals als vorweggenommener Zusatz nach 96,5-6 (Jo 20,13) wyp warvmme weynestú $\mathrm{Fr} \mathrm{Br} \mathrm{N1} \mathrm{N2}$ in der Perikope von der Begegnung Maria Magdalenas mit dem Auferstandenen nach Jo 20,11-18.

Ebensowenig überrascht es, daß die Vulgata auf den verschiedenen Überlieferungsstufen auf den Text einwirkt. Dies läßt sich besonders bei der zweiten Nürnberger Handschrift beobachten.

\footnotetext{
28 Vgl. unten S. $370 \mathrm{f}$.

29 In Br N1 fehlt dieser Zusatz.

30 Statt dessen haben $\mathrm{Br} \mathrm{N} 1$ die reguläre Lukasstelle übersetzt: Die da kauften vnd virkauften. 31 In $\mathrm{Br}$ ist bosen statt virfluchten überliefert.
} 